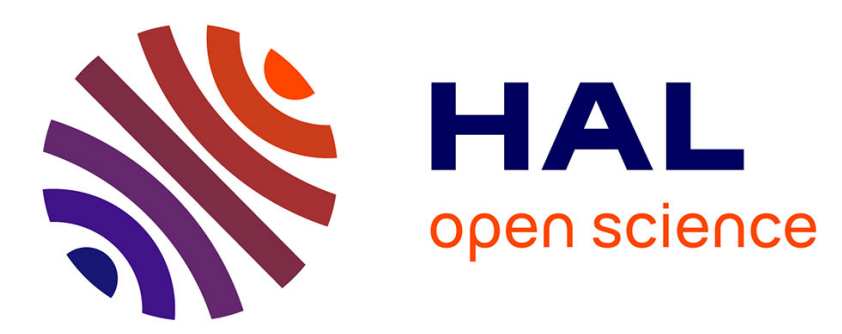

\title{
Joint Routing and Rate Control Scheme for Multi-Stream High-Definition Video Transmission over Wireless Home Networks
}

Liang Zhou, Benoit Geller, Anne Wei, Baoyu Zheng, Jingwu Cui

\section{- To cite this version:}

Liang Zhou, Benoit Geller, Anne Wei, Baoyu Zheng, Jingwu Cui. Joint Routing and Rate Control Scheme for Multi-Stream High-Definition Video Transmission over Wireless Home Networks. Theoretical Computer Science, 2009, 10.1093/comjnl/bxn041 . hal-01225532

\section{HAL Id: hal-01225532 \\ https://hal.science/hal-01225532}

Submitted on 6 Nov 2015

HAL is a multi-disciplinary open access archive for the deposit and dissemination of scientific research documents, whether they are published or not. The documents may come from teaching and research institutions in France or abroad, or from public or private research centers.
L'archive ouverte pluridisciplinaire HAL, est destinée au dépôt et à la diffusion de documents scientifiques de niveau recherche, publiés ou non, émanant des établissements d'enseignement et de recherche français ou étrangers, des laboratoires publics ou privés. 


\title{
Joint Routing and Rate Control Scheme for
} Multi-Stream High-Definition Video

\section{Transmission over Wireless Home Networks}

\author{
Liang Zhou, Benoit Geller, Anne Wei, Baoyu Zheng, Jingwu Cui
}

\begin{abstract}
The support for multiple high-definition video streams in wireless home networks requires appropriate routing and rate control measures ascertaining the set of links for transmitting each stream and the encoding rate of the video to be delivered over the chosen links. The routing and rate allocation procedures impact the sustained quality of each video stream measured as the mean squared error distortion at the receiver, and the overall network congestion in terms of queuing delay per link. We study the trade-off between these two competing objectives in a united convex optimization formulation, and propose a distributed solutions for joint routing and rate control for multiple streams. For each stream, the optimal allocated rate strikes a balance between the selfish motive of minimizing video distortion and the global good of minimizing network congestions, while the routes are chosen over the least-congested links in the network. Simulation results are provided which demonstrate the effectiveness of our proposed joint routing and rate control scheme in the context of wireless home networks.
\end{abstract}

\section{Index Terms}

wireless home networks; routing; rate control; high-definition video

Address: 312 Packard Building, 350 Serra Mall, Stanford University, CA 94305, U.S.A. (Liang Zhou)

Email: zkingzll1981@gmail.com or zkingzll@stanford.edu

Tel: 1-650-723-3731 


\section{ACRONYM AND NOTATION}

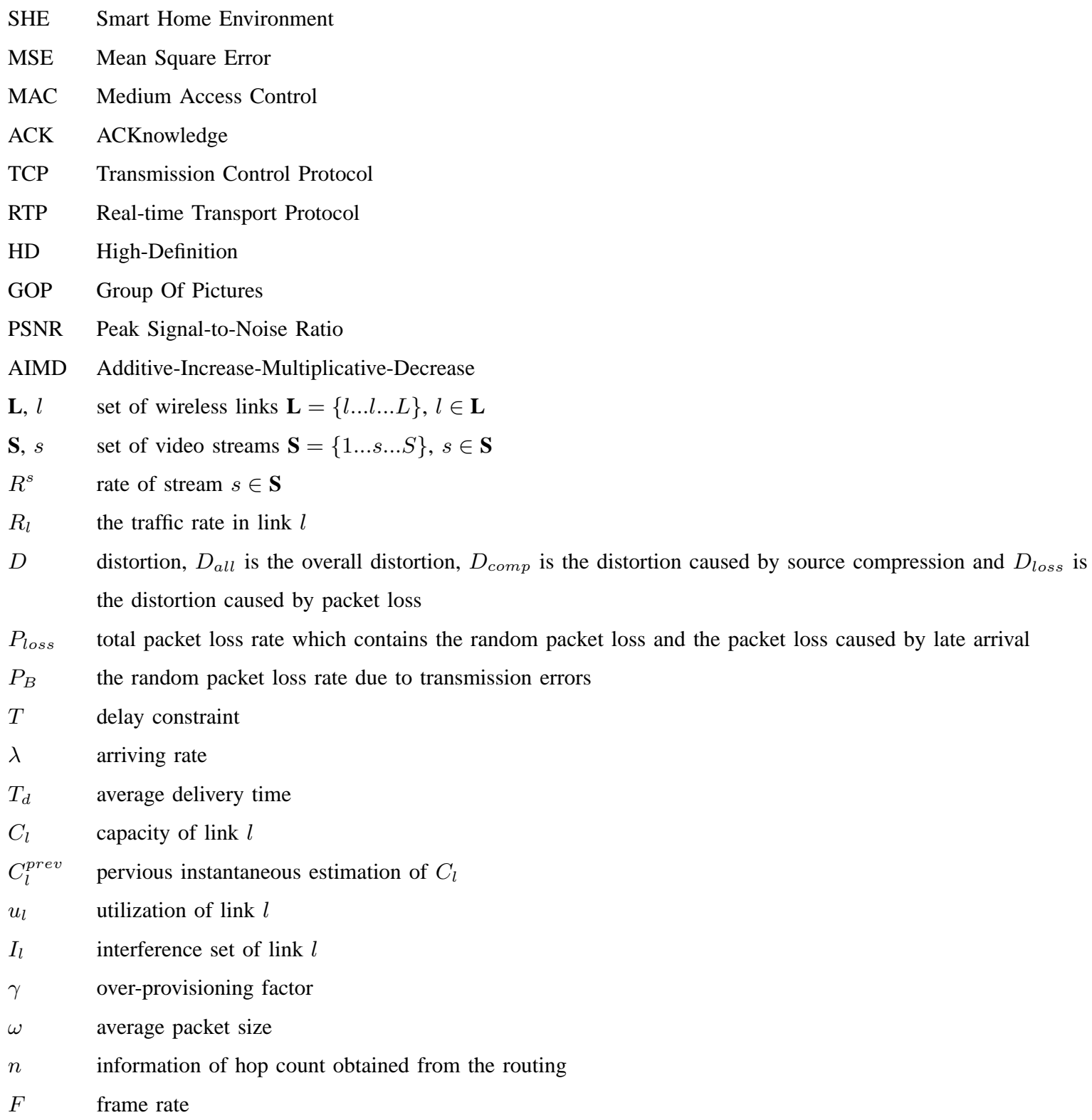

\section{INTRODUCTION}

As multimedia is expected to be a major traffic source on the next-generation wireless networks, supporting multiple High-Definition (HD) video streaming sessions over a wireless home media network has been one of the main fields of attention in the Smart Home Environment 
(SHE) community. Obviously, HD video gives rise to many attractive applications, as well as numerous technical challenges in the SHE. The design of such a system needs to address the unpredictable nature of wireless communication channels, while meeting the high data rate and low latency requirements of media streaming.

Inevitably, there are huge and different kinds of data streaming from different HD video streams which may influence each other and thus, reasonable routing and careful rate control are needed to prevent multiple simultaneous stream sessions from congesting the shared wireless networks. More specifically, the utility of the available routing and allocated rate are also different for streams with different contents: the same rate increase may impact a sequence containing fast motion rather differently than a head-and-shoulder news clip. In addition, it is observed that quality of the received video stream is also affected by the self-inflicted network congestion leading to packet drops due to late arrivals. An attempt to enhance the system performance should therefore account for both metrics in a congestion-distortion optimized fashion. When multiple streams are present in a wireless network, the chosen rate and routes for each stream would also affect the performance of others. Therefore, both rate allocation and routing need to be optimized for all streams in the network, preferably in a distributed manner.

In this paper, we study a convex optimization formulation of the joint routing and rate control problem for multiple HD video sessions sharing a common wireless home network. The main contributions or novelties of this paper consist of: (1) developing a united distortion model which captures both the impact of encoder quantization and packet loss due to network congestion on the overall video quality; (2) proposing a distributed joint routing and rate control scheme, where the allocated rate at each video stream depends on both the distortion-rate characteristic of the video and the network congestion increment, which in turn is obtained from a distributed routing procedure. In order to get a distributed solution of joint routing and rate control, we show that the optimal global trade-off between total video distortion of all streams versus overall network congestion can be translated into the local balance between reducing encoded video distortion versus constraining network congestion at each stream. So, for each stream, the optimal allocated rate strikes a balance between the selfish motive of minimizing video distortion and the global good of minimizing network congestions, while the routes are chosen over the least-congested links in the network.

The rest of paper is organized as follows. In the next section, we formulate the problem 
from the aspects of the video compression and network congestion, respectively. In Section III, we first summarize the congestion-distortion optimized routing, which serves as the basis for the joint routing and rate control scheme in the latter. And then, we provide some selected simulation results for the proposed joint scheme in Section IV. In section V we present related works on routing protocols and rate control methods for multiple streams related to wireless home networks, followed by concluding remarks in Section VI.

\section{Problem Formulation}

In low-latency streaming applications, compressed video is transmitted over a network at a given rate. Typically, it is desirable to achieve end-to-end delays of no more than a few hundred milliseconds. In fact, the reconstructed video quality is affected by both source compression and quality degradation due to packet losses either caused by transmission errors or late arrivals. More specifically, the distortion introduced by quantization is evenly distributed across the encoded frames and is determined by the encoding bit-rate, while packet loss which usually introduces decoding errors lies in the frames containing the missing packets. In this paper, we assume that the two forms of induced distortion are independent and additive. Thus, we can calculate the overall distortion $D_{\text {all }}$ in terms of using MSE (Mean Square Error) as

$$
D_{\text {all }}=D_{\text {comp }}+D_{\text {loss }}
$$

where the distortion introduced by quantization at the decoder is denoted by $D_{\text {comp }}$, and the additional distortion caused by packet loss is denoted by $D_{\text {loss }}$. Considering a set of wireless links $\mathbf{L}$, and a set of video streams denoted by $\mathbf{S}$, for each video stream $s \in \mathbf{S}$, the distortion caused by source compression can be approximated by:

$$
D_{\text {comp }}=\frac{\theta^{s}}{R^{s}-R_{0}^{s}}+D_{0}^{s}
$$

where $R^{s}$ is the rate of the video stream $s \in \mathbf{S}, \theta^{s}, R_{0}^{s}$ and $D_{0}^{s}$ are the parameters of the distortion model which depend on the encoded video sequence as well as on the encoding structure [1]. Using nonlinear regression techniques, these parameters can be estimated from empirical ratedistortion curves obtained by encoding a sequence at different rates. Likewise, the distortion caused by packet loss to decoded video can be modeled by a linear model related to the packet loss rate $P_{\text {loss }}$ :

$$
D_{\text {loss }}=\alpha P_{\text {loss }}
$$


where $\alpha$ depends on parameters related to the compressed video sequence, such as the proportion of intra-coded macro-blocks and the effectiveness of error concealment at the decoder [1]. The packet loss rate $P_{\text {loss }}$ reflects the combined rate of random losses and late arrivals of video packets. In a bandwidth-limited network, this combined loss rate can be further modeled based on the $\mathrm{M} / \mathrm{M} / 1$ queuing model [2]. In this case, the delay distribution of packets over a single link is exponential. Note that, since the end-to-end delay of packet delivery in multi-hop network is dominated by the queuing delay at the bottleneck link, the empirical delay distribution for realistic traffic patterns can still be modeled by an exponential:

$$
\operatorname{Pr}\{\text { Delay }>T\}=e^{-\lambda T}
$$

where $T$ is the delay constraint and $\lambda$ is the arriving rate which is determined by the average delay. Generally, $\lambda$ needs to be determined empirically from end-to-end delay statics over the network [2]. In order to present a general solution for online operation, here we construct a model to approximate the average link delay in a general wireless home network.

At first, it is assumed that at any time instance, only one of the competing links is allowed to transmit, which is similar to the CSMA/CA mechanism used in 802.11 networks. We denote link utilization as the fraction of time occupied by a link $l \in \mathbf{L}$ with traffic rate $R_{l}$ and link capacity $C_{l}$ :

$$
u_{l}=R_{l} / C_{l}
$$

where the value of $R_{l}$ equals to one of the $R^{s}, s \in \mathbf{S}$. The effective $C_{l}$ for an stream $R_{l}$ is measured as:

$$
C_{l}=\varsigma C_{l}^{\text {prev }}+(1-\varsigma) \frac{\omega}{T_{d}}
$$

by logging average packet size $\omega$ and average delivery time $T_{d}$ at the MAC (Medium Access Control) layer, including the overhead of header and ACK (ACKnowledge) transmissions. In (6), $C_{l}^{\text {prev }}$ denotes the pervious instantaneous estimation of $C_{l}$, which is smoothed over time with the value of $\varsigma$ empirically chosen at 0.95 .

The set of links that cannot transmit simultaneously with link $l$ constitutes the interference set of link $l$, denoted as $I_{l}$. In this case, the total utilization is constrained to be smaller than unity:

$$
u_{I_{l}}=\sum_{l \in I_{l}} u_{l}<1
$$


In practice, the total channel time proportion is constrained to be $u_{I_{l}}<\gamma$, where $\gamma<1$ is over-provisioning factor.

Congestion over each wireless link is measured as the average delay for all packets traversing that link. Following the classic M/M/1 queuing model, where average packet delay over a single link is inversely proportional to the residual link capacity, we propose to model the average packet delay for multiple links competing within the same network as ${ }^{1}$ :

$$
E\{\text { Delay }\}=\frac{1}{\lambda}=\sum_{l \in I_{l}} \frac{n \cdot \omega}{C_{l}\left(\gamma-u_{I_{l}}\right)}
$$

where $n$ is the hop count which can be obtained from the routing algorithm at the network layer. Therefore,

$$
\operatorname{Pr}\{\text { Delay }>T\}=e^{-\frac{1}{\sum_{l \in I_{l}} \frac{1 n \cdot \omega}{C_{l}\left(\gamma-u_{I}\right)} T} T}
$$

Together with an average packet loss rate $P_{B}$ due to transmission errors, the total packet loss rate is then:

$$
P_{\text {loss }}=P_{B}+\left(1-P_{B}\right) \operatorname{Pr}\{\text { Delay }>T\}=P_{B}+\left(1-P_{B}\right) e^{-\frac{1}{\left.\sum_{l \in I_{l}} \overline{C_{l}(\gamma \cdot \omega}-u_{I_{l}}\right)} T}
$$

The total distortion from packet loss can be expressed as

$$
D_{\text {loss }}=\alpha P_{\text {loss }}=\alpha\left(P_{B}+\left(1-P_{B}\right) e^{-\frac{1}{\left.\sum_{l \in I_{l}} \overline{C_{l}(\gamma \cdot \omega} u_{I_{l}}\right)} T}\right)
$$

Based on the previous discussion, we seek to minimize the sum of the total distortion $D_{\text {all }}=$ $D_{\text {comp }}+D_{\text {loss }}$ as follows:

$$
\min _{R^{s}, s \in S} \sum_{s \in S} \frac{\theta^{s}}{R^{s}-R_{0}^{s}}+D_{0}^{s}+\alpha\left(P_{B}+\left(1-P_{B}\right) e^{-\frac{1}{\sum_{l \in I_{l}} \frac{1, \cdot \omega}{C_{l}\left(\gamma-u_{I_{l}}\right)}} T}\right)
$$

Intuitively, reconstructed video quality is limited by coarse quantization at lower rates; whereas at high rates, the video stream will cause more network congestion. This, in turn, translates into higher loss rates, hence reduces video quality. For live video steaming in bandwidth-limited environments, we therefore expect to achieve maximum decoded quality for some intermediate rate.

\footnotetext{
${ }^{1}$ In practice, congestion may be a more complicated function of rate as predicted by $\mathrm{M} / \mathrm{M} / 1$ model. However, this expression can be viewed as an approximation of the average link delay, capturing the non-linear increase of delay with total channel time utilization.
} 


\section{Joint Routing And RATE CONTROL Scheme}

In this section, we address the problem of joint routing and rate control among multi-stream HD video transmission over wireless networks. At first, we show route selection and rate control for each stream can be jointly optimized to trade-off between overall network congestion and total video distortion of all streams.

\section{A. Congestion-Optimized Multi-Path Routing}

Consider a simpler special case of the problem formulated in (12), where only one video stream is involved, and its rate $R^{s}$ is fixed. This then becomes the classical problem of minimizing network congestion via optimal flow assignment. Some centralized routing and traffic partitioning algorithms have been proposed for video over wireless ad-hoc networks, where multiple routes are extracted from the optimal flow assignment result, and the total traffic is dispersed over the multiple paths in a congestion-optimized manner [5].

This centralized scheme, however, requires knowledge of global network information such as capacities and flows along all the links, which restricts the scalability of the network. The computational complexity of the optimization and route extraction from the flow assignment may also exceed the capabilities of any single node in the network. To counter the problem, the total rate of the video stream $R^{s}$ is split into $K$ small increments such that $R^{s}=\sum_{k=1}^{K} \triangle R_{k}^{s}$. Then the optimal allocation of increment $\triangle R_{k}^{s}$ can be achieved by finding a path $P_{k}^{*}$ that accomplishes the following:

$$
\min _{P_{k}^{*}} \sum_{l \in L} \frac{C_{l}}{C_{l}-R_{l}^{\prime}} \triangle R_{k}^{s}
$$

where $R_{l}^{\prime}=R_{l}+\sum_{l^{\prime} \in I_{l}} R_{l^{\prime}}$ includes current traffic $R_{l}$ and other competing traffics for link $l$. Note that the minimization objective in (13) corresponds to end-to-end accumulated sum of $C_{l} /\left(C_{l}-R_{l}^{\prime}\right)$, which can be interpreted as "congestion sensitivity" over that link (i.e., amount of increase in congestion per unit increase in rate). Consequently, the optimal path $P_{k}^{*}$ in (13) can be found via the distributed Bellman-Ford algorithm [6], by setting the link cost to congestion sensitivity. Every node maintains a minimum-cost path from itself to the source, exchanges this information with its neighbors, and updates to a lower cost path if it discovers one via a neighbor. When the destination node reports the chosen path for a given rate increment $\triangle R_{k}^{s}$ to the sender, it can easily append the corresponding accumulated congestion sensitivity value to 
the path information, for later use in the rate control process as explained in the next section. The reader is referred to the original paper for further details of the distributed routing protocol.

\section{B. Distributed Joint Routing and Rate Control}

Here, we discuss the framework for a distributed methodology to allocate rate to a video stream $s \in \mathbf{S}$. The Karush-Kuhn-Tucker (KKT) necessary and sufficient conditions for the optimal solution to (12) state that the allocated rate to stream $s$ should either meet the boundary condition exactly, or correspond to zero partial derivative:

$$
\frac{d D_{\text {comp }}}{d R^{s}}+\alpha \frac{d D_{\text {loss }}}{d R^{s}}=0
$$

where $d D_{\text {comp }} / d R^{s}$ is derived from the video distortion model (12) as

$$
\frac{d D_{\text {comp }}}{d R^{s}}=-\frac{\theta^{s}}{\left(R^{s}-R_{0}^{s}\right)^{2}}
$$

Hence the distortion reduction caused by increasing encoding rate by $\triangle R_{k}^{s}$ is

$$
-\triangle D_{\text {comp }}^{k} \approx \frac{\theta^{s}}{\left(R^{s}-R_{0}^{s}\right)^{2}} \triangle R_{k}^{s}
$$

The slope of packet loss distortion increment $d D_{\text {loss }} / d R^{s}$, on the other hand, can be expressed as:

$$
\frac{d D_{\text {loss }}}{d R^{s}} \propto\left(1-P_{B}\right) \sum_{l \in L} \frac{1}{\gamma-u_{I_{l}}} \approx\left(1-P_{B}\right) \sum_{l \in L} \frac{1}{1-\sum_{l^{\prime} \in I_{l}} u_{l^{\prime}}}
$$

The resulting packet loss distortion increment $\Delta D_{\text {loss }}^{k}$ can be then be approximated as:

$$
\triangle D_{\text {loss }}^{k} \approx\left(1-P_{B}\right) \sum_{l \in L} \frac{C_{l}}{C_{l}-R_{l}^{\prime}} \Delta R_{k}^{s}
$$

where cross-traffic $R_{l}^{\prime}$ includes contributions from current traffic $R_{l}$ and other video streams. Note that (18) is almost the same as the optimization criterion in (13), and can be accumulated over the chosen links of path. This information is then collected at the destination node and fed back to the source.

Given the packet loss distortion increment $\Delta D_{\text {loss }}^{k}$ in (18) and the video compression distortion reduction $-\triangle D_{\text {comp }}^{k}$ in (16), the source node can make the rate control decision by comparing the two quantities. The allocated rate will be increased by $\Delta R_{k}^{s}$ until $-\Delta D_{\text {comp }}^{k}>\alpha \Delta D_{\text {loss }}^{k}$, i.e., when the benefit of distortion reduction is no longer worthwhile the consequential network congestion. Due to the convex nature of both $D_{\text {comp }}$ and $D_{\text {loss }}$, the initial distortion reduction 


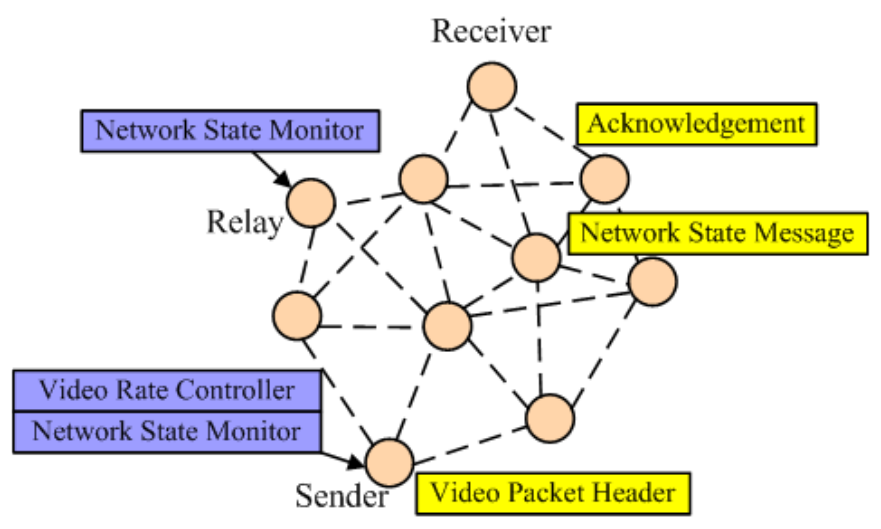

Fig. 1. Cross-layer information exchange among the network state monitor at the network layer and the video rate controller at the transport layer.

is typically significant for small rate increments, whereas increase in network congestion starts out slowly. Therefore, the rate control algorithm can continue until it reaches the optimal rate that strikes a balance between the two trade-off slopes.

In order that the source rates can be adapted at the transport layer according to network states reported from the network layer, the cross-layer information exchange is needed. Fig.1 illustrates various components in such a system. At the MAC layer, a link state monitor keeps an online estimate of the effective capacity $C_{l}$. It also records the intended rate allocation $R^{s}$ advertised by each stream, and calculates the link utilization $u_{l}$ accordingly. Periodic broadcast of link state messages are used to collect the values of $C_{l}$ and $u_{l}$ from neighboring links in the same interference set. At the network layer, the routing information obtained from the routing algorithm can be used to calculate the $P_{\text {loss }}$. At the application layer, the video rate controller at the source advertises its intended rate control $R^{s}$, and calculates the value of $D_{\text {all }}$ accordingly. The link state monitor traversed by the stream then calculates the relevant parameters in (12) based on its local cache of capacity, utilization information of all the links within its interference set. The destination node extracts such information from the video packet header and reports back to the sender in the acknowledgment packets, so that the video rate controller can re-optimize its intended rate $R^{s}$ based on the proposed joint routing and rate algorithm, with updated link state information. 


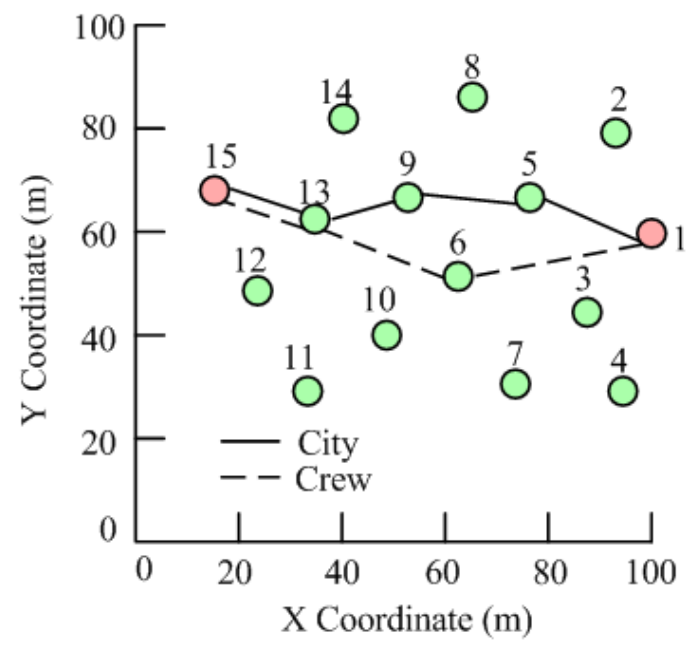

Fig. 2. Example network with 15 nodes randomly positioned in a $100 \mathrm{~m}$-by-100m square area. Both of the video streams are sent from node 1 to node 15 .

\section{Simulation Results AND Discussions}

In this section, we conduct simulation experiments to study the performance of the proposed joint routing and rate control scheme in a distributed framework, in which we employ 802.11a network as a wireless home network. At first, we describe the simulation environment. And then, we present the main simulation results where we show the objective results of the performance of the proposed scheme under different scenarios. Finally, we conclude this section by summarizing the conclusions based on the selected simulation results.

\section{A. Simulation Setting}

We simulate a small wireless network with 15 nodes randomly placed in a $100 \mathrm{~m}-\mathrm{by}-100 \mathrm{~m}$ square in ns-2, all with transmission range of each other, as illustrated in Fig.2. Each node follows the IEEE 802.11 a protocol, with a rate of $54 \mathrm{Mbps}$ for playload and $6 \mathrm{Mbps}$ for MAC headers and ACK packets. Here, we use a two-state Markov model (i.e.Gilbert model) to simulate the bursty packet loss behavior. The two states of this model are denoted as G (Good) and B (Bad). In state $\mathrm{G}$, packets are received correctly and timely, whereas, in state B, packets are assumed to be lost. This model can be described by the transition probabilities $P_{G B}$ from state $\mathrm{G}$ to $\mathrm{B}$ 


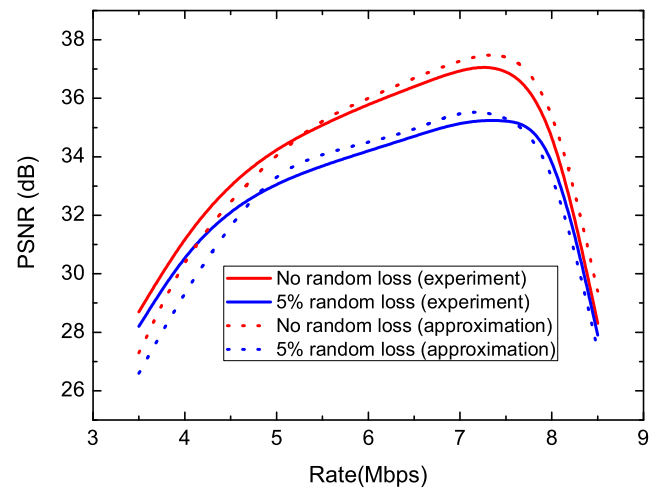

(a) City

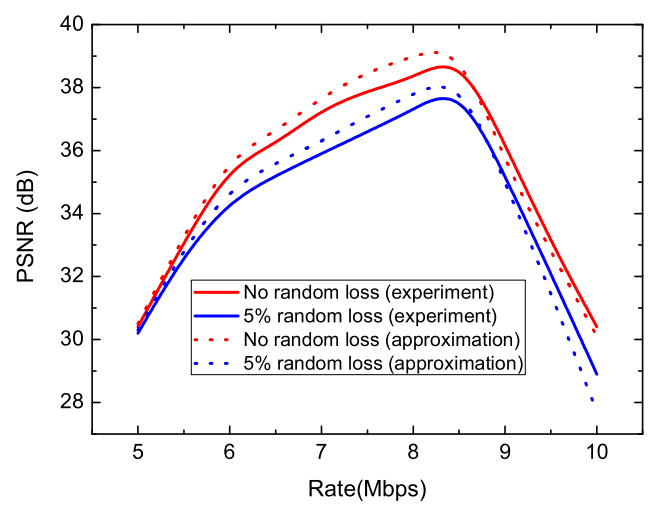

(b) Crew

Fig. 3. Decoded video quality approximate model and experimental data for HD City and Crew sequences at 60 frames per second and GOP length of 16 and a playout deadline of $350 \mathrm{~ms}$. The value of $\alpha$ is 202 for both sequences.

and $P_{B G}$ from state $\mathrm{B}$ to $\mathrm{G}^{2}$.

Two HD video sequences City and Crew, of varying content complexity, are considered for streaming over wireless networks. The sequences have spatial resolution of $1280 \times 720$ pixels, and frame rate of $60 \mathrm{fps}$. The video sequences are encoded using the H.264/AVC reference codec JM10.2 [7], with GOP (Group Of Pictures) length of 16 and IBBP... structure. Each slice is constrained to have maximum size of 1400 bytes, and fits into one RTP (Real-time Transport Protocol) packet. For each pruned version of the encoded bitstream, packet transmission intervals are spread out evenly in the entire GOP duration, so as to avoid unnecessary bursts due to large I frames. Playout deadline is chosen to be $350 \mathrm{~ms}$. Since small ACK packets incur much MAClayer overheads during transmission, only one ACK is sent per ten received packets.

\section{B. Performance Evaluation of the Proposed Scheme}

At first, we validate the distortion model introduced in Section II. Fig.3 shows the rate-PSNR (Peak Signal-to-Noise Ratio) tradeoff when HD City and Crew (300 frames) stream over the

\footnotetext{
${ }^{2}$ The state transitional probabilities $P_{G B}$ and $P_{B G}$ are fitted to a 15 -second packet delivery trace collected in [4], with MAClevel packet loss ratio of $8.3 \%$, average duration of $0.8 \mathrm{~ms}$ for the bad state, and $8.8 \mathrm{~ms}$ for the good state. We then choose similar state transitional probability values, to simulate channels with MAC-level loss ratios in the range of 3-14\%
} 


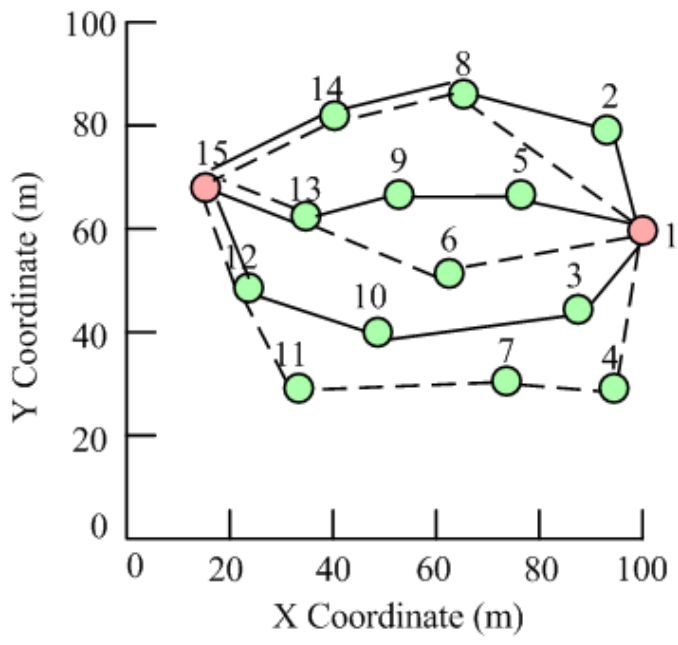

(a)

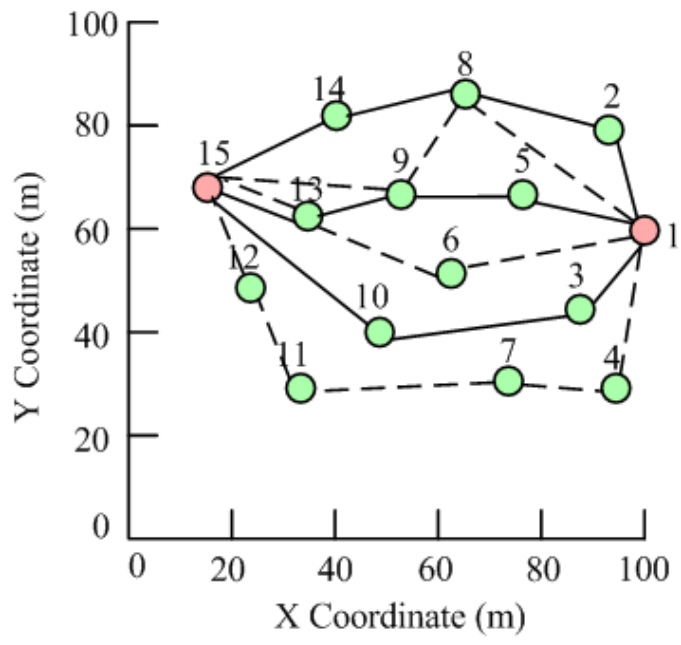

(b)

Fig. 4. (a)Initial and (b) final route selections of all two streams: City in solid line and Crew in dashed line. For this instance, the rate increment is $500 \mathrm{kbps}$.

wireless network, respectively. The two sequences are sent following the routing illustrated in Fig.2. The model is fit to experimental data for two cases: in the first case, the only losses considered are due to late arrivals; in the second, an additional end-to-end random loss rate of $5 \%$ is considered. The bell-shape of the curves illustrates that the highest performance is obtained when the streaming rate achieves the optimal tradeoff between compression quality and self-inflicted congestion. The approximate optimal operating rate computed by numerically solving (12) matches closely with experimental data.

We next evaluate the performance of the proposed optimization measures with multiple video streams. Note that if the chosen routes for each stream travel over non-overlapping links, then the network can be decomposed into independent subsets supporting each stream unaffected by others, which reduces to the scenario of single stream. In order to investigate the interactions among multiple streams, we choose the same source and destination nodes for all streams so as to have shared links in the chosen routes. Fig.4 illustrates the initial and final routes chosen for all two streams during the optimization of the joint routing and rate allocation process. The corresponding rate allocated to each stream is shown in Fig.5 over iteration steps. It can be observed that the selected routes for two of the streams (City in solid line and Crew in dashed line) have both changed over the iterations, each re-dispensing its own traffic over the network 


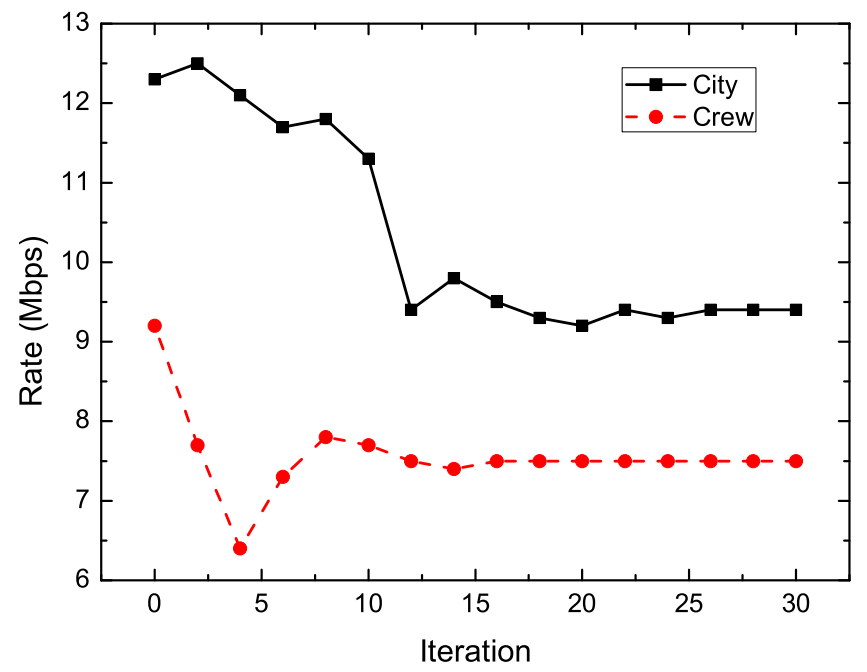

Fig. 5. Allocated rate for each stream over the iterations, corresponding to the same setting in Fig.4

to avoid already congested links. Changes in the routes also affect the congestion increment information calculated during routing, which in turn leads to changes in the rate allocation decisions.

To demonstrate the effectiveness of our proposed rate control algorithm, we use the representative drop-tail scheme which employs the fixed rate allocation and the Additive-IncreaseMultiplicative-Decrease (AIMD)-based rate control method which is used by TCP (Transmission Control Protocol) congestion control [3] for comparison. In order to get a more clear picture of how the allocated rate reflects the reconstructed quality, we just use one test sequence, city, streaming over network in this simulation. More specifically, the drop-tail scheme employs a fixed source coding rate $R_{f}=8 \mathrm{Mbps}$ and when the rate exceeds the current optimal transmission rate available for the selected source-destination pair, it will drop the subsequent encoded packets. Instead of performing proactive rate controls by optimizing a chosen objective according to observed network and video characteristics, the AIMD-based scheme is reactive in nature, probing the network for available bandwidth and reducing rate control after congestion occurs. Each stream $s$ initiates its rate at a specified rate $R_{s}^{A I M D}$ corresponding to the minimum acceptable video quality, and increases its allocation by $\Delta R_{s}$ every $\Delta t$ seconds unless network 


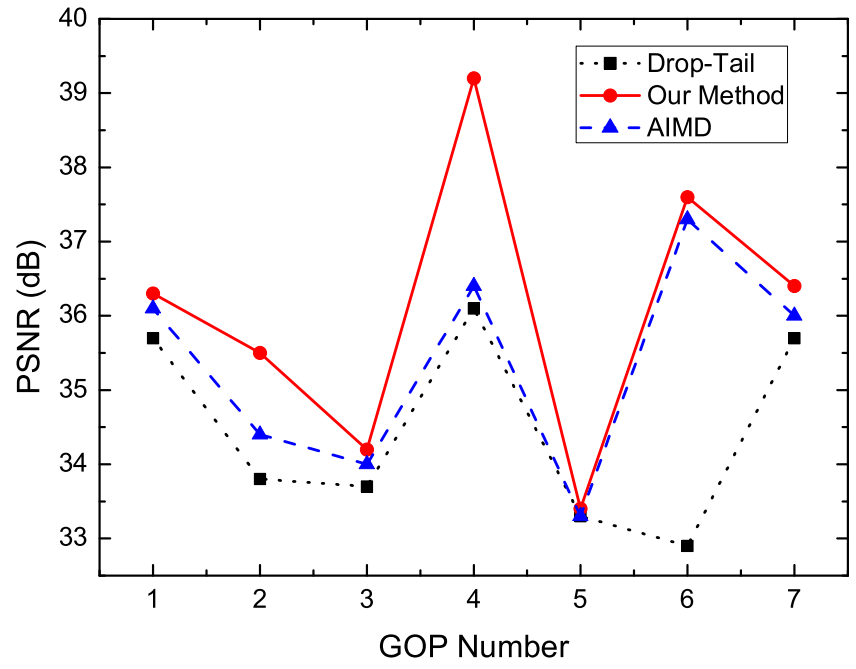

(a)

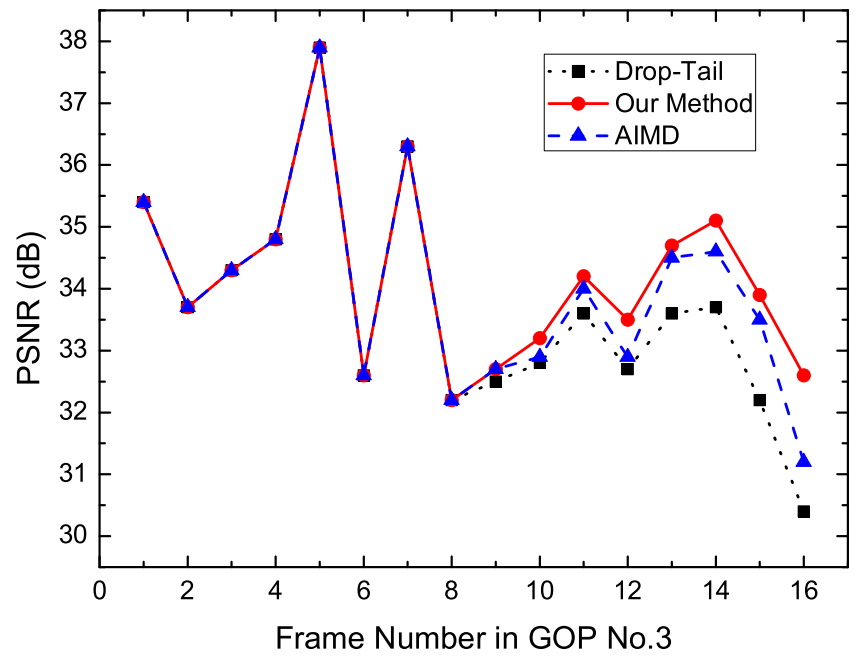

(b)

Fig. 6. Performance comparison between different rate control schemes. 
TABLE I

Corresponding Channel Profile to Fig.6(MbPs)

\begin{tabular}{|c|c|c|c|c|c|c|c|}
\hline GOP No. & 1 & 2 & 3 & 4 & 5 & 6 & 7 \\
\hline$R$ (Our Method) & 9.2 & 10.4 & 6.7 & 12.3 & 7.9 & 14.5 & 6.1 \\
\hline$R$ (AIMD) & 8.9 & 9.3 & 7.0 & 8.4 & 8.0 & 14.1 & 6.9 \\
\hline$R_{f}$ & 8.0 & 8.0 & 8.0 & 8.0 & 8.0 & 8.0 & 8.0 \\
\hline
\end{tabular}

congestion is perceived, in which case the allocated rate is dropped by $\left(R^{s}-R_{s}^{A I M D} / 2\right)$.

In Fig.6, we show a performance comparison between our proposed rate control scheme and the competing methods, drop-tail and AIMD, in the scenario where packet losses are caused only by channel over-pumping ${ }^{3}$. In Fig.6(a), the average PSNR using the proposed rate control algorithm is $36.08 \mathrm{~dB}$ while it is 35.39 using AIMD-based method and $34.46 \mathrm{~dB}$ for the case of drop-tail. Thus, using our proposed algorithm can achieve almost $0.69 \mathrm{~dB}$ and $1.62 \mathrm{~dB}$ performance gains comparing to the AIMD and drop-tail scheme, respectively. From the network profile, illustrated in Table I (the value is averaged over one GOP), we can see that for GOP No.1, No.2, No.4, and No.6, the operation transmission rate is higher than the fixed $8 \mathrm{Mbps}$. Thus, using our rate control can fully exploit the optimal transmission rate resulting in improved performance compared to using a fixed-rate coding scheme. On the other hand, for GOP No.3, No.5, and No.7, it is obvious that the fixed source coding rate is higher than the operation transmission rate; therefore, packet losses will occur when the transmission buffer is full resulting in the last couple of frames being lost which cause substantial performance degradation. A lost frame is concealed by just copying the previous frame and if several consecutive frames are lost, the degradation will be even more serious since the concealed frames are then used as correctly received frames to conceal the subsequent lost frames. This results in substantial error propagation which can obviously observe from Fig.6(b) which presents the each frame PSNR value in GOP No.3. It should also be noted from Fig.6 that the performance achieved by the proposed rate control method is also super to the traditional AIMD-based method. On one hand, although the AIMDbased method can adapt to the network condition, the network is so dynamic that a congested

\footnotetext{
${ }^{3}$ Here, we assume that no transmission errors occurred
} 
node forwarding a few seconds might not be used at all at the point in time when the source reacts to the congestion. On the other hand, the proposed rate control method further take advantage of explicit knowledge of the video distortion-rate characteristics, and can achieve more balanced video quality.

\section{Observations}

Based on the selected objective simulation results described above, there are several main observations:

- The routing algorithm is a basic facet in implementing the video transmission over wireless networks. The proposed routing algorithm can re-dispensing the traffic to avoid already congested links.

- The rate control plays an important role in the whole video transmission system. If the operating rate is lower than the optimal transmission rate, performance loss is due to the source coding inefficiency resulting from the use of an unnecessarily lower source coding rate; if the operating rate is higher, performance loss is caused by packet losses due to buffer overflow and network congestion.

- The joint routing and rate control scheme has the effectiveness in achieving the optimal congestion-distortion trade-off for the overall system.

\section{RELATED WORKS}

In this section, we present the related works on the ad-hoc routing protocols and multi-stream rate control algorithm related to the wireless home networks. In addition, we also indicate the difference between our proposed routing and rate control with the previous works.

\section{A. Ad-Hoc Routing Protocols}

Several ad-hoc routing protocols that have been proposed over the years include proactive tabledriven protocols like Destination-Sequenced Distance-Vector (DSDV) routing [8] and Optimized Link State Routing (OLSR) [9], as well as on-demand protocols like Dynamic Source Routing (DSR) [10] and Ad-hoc On-demand Distance Vector (AODV) routing [11]. The former involve the evaluation and storage of the routing tables pertaining to the topology at each node. The routing tables are periodically updated to counter the topological changes associated with 
node mobility and wireless channel variations. This can result in significant protocol overhead, especially under high node mobility and dynamic channel conditions. The on-demand protocols on the other hand involve discovery of the route whenever data need to be transmitted between a source-destination pair. They typically incur less overhead traffic than the table-driven protocols, and can consequently better adapt to dynamically varying topologies. Comparative study of various routing protocols has been an active research area in the wireless ad-hoc networking community.

The aforementioned routing strategies evaluate the best sequence of nodes in accordance with criteria like minimum hop or delay, and forward data along a single path. Couto et al. [12] pointed out the inadequacy of minimum-hop routing in wireless ad-hoc networks, and proposed alternative link metrics for evaluating a path. Extensions to multi-path routing have also been proposed for multi-path AODV [13] and for ExOR, an opportunistic multi-hop routing strategy that broadcasts data packets to explore multiple paths in the network [15]. For video streaming, benefits of multi-path routing over ad-hoc networks are demonstrated in terms of robust packet delivery via path diversity and higher sustainable rate and quality due to bandwidth aggregation [16].

Unlike most previous works that consider routing for generic data traffic over wireless networks, we take into account specific characteristics of video streaming in the evaluation of routes. Network congestion is incorporated explicitly into the route selection metric, to meet the stringent latency requirement for video packet delivery. The rate-distortion characteristic of each stream is also considered in the rate allocation procedure to accommodate multiple streams with various video contents and complexity.

\section{B. Multi-Stream Rate Allocation}

The issue of multi-stream rate allocation is still an open problem and has received considerable attention recently. Practical solutions such as TCP congestion control [18], [3] and TCP-Friendly Rate Control (TFRC) [14] are widely used over the Internet. A mathematical framework of multi-user rate allocation is presented in [19], where the authors also analyzed two classes of distributed solutions, corresponding to the primal and dual decomposition of the optimization objective. In wireless networks, adaptive transmission techniques are typically used to protect the video stream against the time-varying channel [20]. When multiple streams are involved, 
centralized channel time allocation among multiple wireless stations has been investigated in [21]. Distributed algorithms have also been proposed, using rate-distortion optimized packet scheduling in [22] for rate allocation among streams sharing a bottleneck link, and using the subgradient method in [17] for streams competing over a wireless mesh network.

For a more practical setting, a rate allocation algorithm combined with a packet partitioning algorithm has been proposed to support video streaming from multiple senders to a single receiver over the Internet [24]. The rates are chosen to match the available network bandwidth for each stream, and the packet partitioning is designed to minimize start up delay. For video streaming over a wireless hop, a rate control scheme has been shown to efficiently utilize the available wireless link capacity using multiple TFRC connections [23].

Our approach targets rate control in conjunction with route selection. The optimization objective function is comprised of both video distortion and network congestion. This differs from most existing works where routing and rate allocation are performed separately, without the notion of limiting overall network congestion.

\section{CONCLUding REMARKS}

In this paper, we use cross-layer design to maximize the perceived wireless video quality by combing routing and rate control techniques. As detailed in the paper, our proposed joint scheme can adaptively to dynamic network condition by adjust the routing and the allocated rate for each video stream. The simulation results demonstrate the effectiveness of our proposed joint routing and rate control scheme for multi-stream high-definition video transmission over wireless home networks.

\section{ACKNOWLEDGMENTS}

This work is partially supported by NSF under Grant 0430886 and the International Project PRA-SI (financed by France and China governments) under Grant No. SI04-03. Moreover, we also thank the anonymous reviewers for insightful comments and suggestions. 


\section{REFERENCES}

[1] K. Stuhlmuller, N.Farber, M.Link and B.Girod, "Analysis of video transmission over lossy channels", IEEE Jounal Select. Areas Commun., vol.18, no.6, pp. 1012-1032, 2000.

[2] X. Zhu, E. Setton and B. Girod, "Congestion-distortion optimized video transmission over ad hoc networks", Journal of Signal Processing: Image Communication, vol. 20, pp. 773-783, 2005.

[3] E. Altman, K. Avrachenkov, et al., "Performance analysis of AIMD mechanisms over a multi-state Markovian path", Computer Networks, vol.47, no.3, pp.307-326, 2005.

[4] P. van Beek and M. Umut Demircin, "Delay-constrained rate adaptation for robust video transmission over home networks", Proc. IEEE International Conference on Image Processing (ICIP05), Genoa, Italy, vol. 2, pp. 173-176, 2005.

[5] E. Setton, T. Yoo, X. Zhu, A. Goldsmith and B. Girod, "Cross-layer Design of Ad Hoc Networks for Real-Time Video Streaming”, IEEE Wireless Communications Magazine, vol. 12, no. 4, pp. 59-65, August 2005.

[6] S. Boyd, L. Vandenberghe, "Convex Optimization”, Cambridge Universtity Press, pp. 76-88, 2004, United Kingdom.

[7] “JM 10.2.”, http://iphome.hhi.de/suehring/tml.

[8] C.E. Perkins, P. Bhagwat, "Highly Dynamic Destination- Sequenced Distance-Vector Routing (DSDV) for Moblie Computers", Proc. ACM Conference on Communications Architectures, Protocols and Applications (SIGCOMM' 94). London, United Kingdom, pp.234-244, 1994.

[9] T. Clausen, P. Jacquest, “Optimized Link State Routing Protocol (OLSR)”, RFC 3626, 2003.

[10] D.B. Johnson, D.B. Maltz, “Dynamic Source Routing in Ad hoc Wireless Networks. Mobile Computing”, Kluwer Academic Publishers, 1996.

[11] C. Perkins, E. Royer, S. Das, “Ad-hoc On-Demand Distance Vector (AODV) Routing”, RFC 3561, 2003.

[12] D.S.J. Couto, D. Aguayo, J. Bicket, R. Morris, "A High-Throughput Path Metric for Multi-Hop Wireless Routing”, Proc. ACM Ninth International Conference on Mobile Computing and Networking (MOBICOM03), San Diego, California, USA, 2003.

[13] M.K. Marina, S.R. Das, “On Demand Multi-Path Distance Vector Routing in Ad hoc Networks”, Proc. IEEE International Conference on Network Protocols, Riverside, California, USA, pp.14-23, 2001.

[14] S. Floyd and K. Fall, "Promoting the use of end-to-end congestion control in the Internet", IEEE/ACM Trans. on Networking, vol. 7, pp. 458-472, Aug. 1999.

[15] S.Biswas, R. Morris, "ExOR: Opportunistic Multi-Hop Routing for Wireless Networks", Proc. ACM Conference on Communications Architectures, Protocols and Applications (SIGCOMM05), Philadelphia, Pennsylvania, USA, pp.133-144, 2005.

[16] D. Li and Q. Zhang, "Multi-source multi-path video streaming over wireless mesh networks", in IEEE ISCAS 2006.

[17] X. Zhu and B. Girod, "Distributed rate allocation for multistream video transmission over ad hoc networks," Proc. IEEE International Conference on Image Processing (ICIP05), Genoa, Italy, vol. 2, pp. 157-160, Dec. 2005.

[18] V. Jacobson, "Congestion avoidence and control", Proc. SIGCOMM 88, vol. 18, no. 4, Aug. 1988.

[19] F. Kelly, A. Maulloo, and D. Tan, "Rate control for communication networks: Shadow prices, proportional fairness and stability,” Journal of Operations Research Society, vol. 49, no. 3, pp. 237-252, 1998.

[20] Y. Shen, P. C. Cosman, and L. B. Milstein, "Error resilient video communications over CDMA networks with a bandwidth constraint,” IEEE Trans. on Image Processing, vol. 15, no. 11, pp. 3241-3252, Nov. 2006.

[21] M. van der Schaar and N. Sai Shankar, "Cross-layer wireless multimedia transmission: challenges, principles, and new paradigms," IEEE Wireless Communications, vol. 12, no. 4, pp. 50-58, Aug. 2005. 
[22] J. Chakareski and P. Frossard, "Rate-distortion optimized distributed packet scheduling of multiple video streams over shared communication resources," IEEE Trans. on Multimedia, vol. 8, no. 2, pp. 207-218, Apr. 2006.

[23] M. Chen, A. Zakhor, "Rate Contcrol for Streaming Video over Wireless", Proc. Twenty-third Annual Joint Conference of the IEEE Computer and Communications Societies (INFOCOM04), Hong Kong, China, pp.1181-1190, 2004.

[24] T. Nguyen, A. Zakhor, "Multiple sender distributed video streaming”, IEEE Trans. on Multimedia, vol.6, no.2, pp.315-326, 2004. 